\title{
On the assembly and calibration of a spatial heterodyne interferometer for limb sounding of the middle atmosphere
}

\author{
Martin Kaufmann ${ }^{1,2}$ (1) Friedhelm Olschewski ${ }^{2} \cdot$ Klaus Mantel $^{3}$ - Oliver Wroblowski ${ }^{1}$ Q Qiuyu Chen ${ }^{1} \cdot$ Jilin Liu $^{1}$. \\ Qiuchen Gong ${ }^{1} \cdot$ Daikan Wei $^{1} \cdot$ Yajun Zhu ${ }^{1} \cdot$ Tom Neubert $^{4} \cdot$ Heinz Rongen $^{4} \cdot$ Ralf Koppmann $^{2} \cdot$ Martin Riese $^{1,2}$
}

Received: 14 April 2019 / Revised: 17 June 2019 / Accepted: 20 June 2019 / Published online: 23 July 2019

(c) The Author(s) 2019

\begin{abstract}
Spatial heterodyne interferometers are an enabling technology to build highly miniaturized optical instruments for the observation of faint emissions in the atmosphere. They are particularly suited for the deployment on nano- or micro-satellite constellations. One application of the SHI technology is a middle atmosphere temperature sounder based on the measurement of relative intensities emitted by the $\mathrm{O}_{2}$ atmospheric band system. Beside basic design considerations, aspects of the opto-mechanical design and assembly of a monolithic SHI for a space application are addressed. For the characterization of such an instrument, a light stimulus based on a Köhler illuminator is presented.
\end{abstract}

Keywords SHS $\cdot$ Spatial heterodyne interferometer $\cdot$ Limb sounding $\cdot$ Atmospheric science $\cdot$ Temperature sounding

\section{Introduction}

For the study of faint signals, Fourier Transform Spectrometers (FTS) have significant advantages over conventional grating spectrometers. Their throughput is typically more than two orders of magnitude larger than grating spectrometers of the same size can deliver. A Spatial Heterodyne Interferometer (SHI) is a special type of an FTS. It has no moving parts and can be built monolithic. Combined with two-dimensional imaging detectors, it can record the interferogram of the scene in one dimension and spatial information in the second dimension.

An SHI can be designed to deliver vertical profiles of temperature in the middle atmosphere without the need of

Martin Kaufmann

m.kaufmann@fz-juelich.de

1 Institute of Energy and Climate Research (IEK-7), Forschungszentrum Jülich, 52425 Jülich, Germany

2 Institute for Atmospheric and Environmental Research, University of Wuppertal, Gausstr. 20, 42119 Wuppertal, Germany

3 Max Planck Institute for the Science of Light, Staudtstr. 2, 91058 Erlangen, Germany

4 Central Institute for Engineering, Electronics and Analytics, Electronic Systems (ZEA-2), Forschungszentrum Jülich, 52425 Jülich, Germany a radiometric calibration. This is possible by observing the relative intensity distribution of emission lines within the $\mathrm{O}_{2}$ $(0,0)$ atmospheric A-band at $762 \mathrm{~nm}[22,25]$. The emitting states have a lifetime of $12 \mathrm{~s}$, which is sufficiently long-lived to assure that the rotational distribution is fully thermalized and can be described by the kinetic temperature. Therefore, by measuring the relative distribution of those lines, atmospheric temperature can be obtained. Figure 1 illustrates the broadening of the A-band emission with increasing temperature. As a broad guide, a $1 \mathrm{~K}$ temperature change affects spectral intensity ratios by $1 \%$ [17]. Typical integration times to obtain an entire altitude profile of temperature are in the order of $1 \mathrm{~s}$ for daytime and $20 \mathrm{~s}$ for nighttime conditions for an instrument of a volume of 31 . A concept for such an instrument compatible with a 6-unit CubeSat was recently presented by Kaufmann et al. [17], Olschewski et al. [21] and references cited therein.

With a constellation of those instruments, it is possible to sense atmospheric waves and their interaction with the global circulation (e.g., polar vortices) with unprecedented spatial and temporal resolution. This will allow to quantify waves coupling different layers of the atmosphere. These waves have a wide range of horizontal scales extending from mesoscale (gravity waves) to global scale (tides, planetary waves). Understanding how energy and momentum are transferred through the generation, propagation, and dissipation of atmospheric waves over a wide range of spatial 


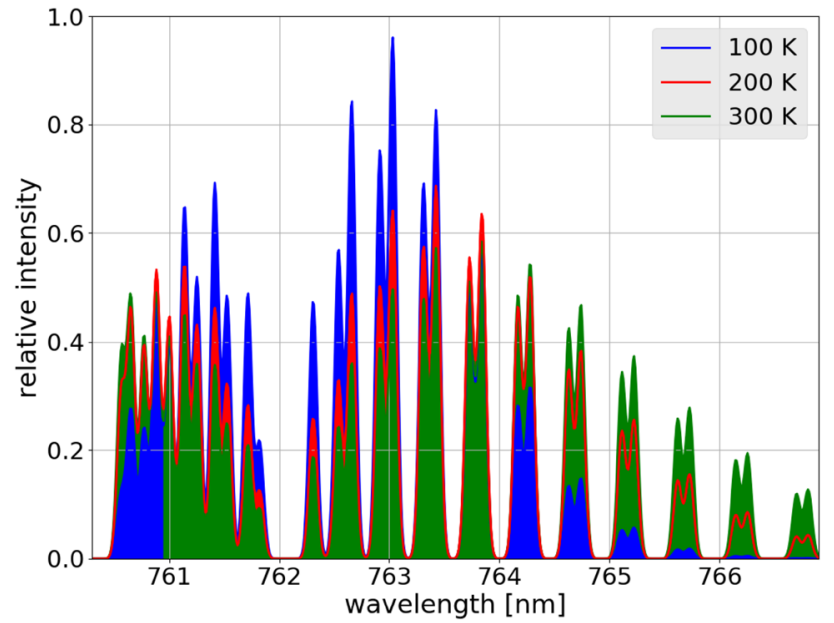

Fig. 1 Simulated $\mathrm{O}_{2}$ A-band spectra at $100 \mathrm{~K}, 200 \mathrm{~K}$, and $300 \mathrm{~K}$, respectively

and temporal scales is one of key factors to understand the variability of the T/I system [20]. Connecting the dynamics of the neutral atmosphere and the ionosphere improves the modelling and forecasting capabilities (see, e.g., [13, 19, $20,24]$. Observing gravity waves in the middle atmosphere at high spatial resolution will also contribute to improving global and regional climate projections and sub-seasonal weather forecasts (10-30 days) due to the downward coupling of these waves.
The enabling technical innovation to measure these waves at the required resolution is highly miniaturized SHI instruments, which are small and powerful enough to be accommodated by nano- or micro-satellites forming a satellite constellation. The second technical innovation is the development of three-dimensional tomographic reconstruction techniques for limb sounding of the atmosphere [16, $27,29]$, which are nowadays sufficiently efficient to process three-dimensional satellite observations in real time.

\section{Basic theory of spatial heterodyne interferometers}

In principle, an SHI is an FTS, where the mirrors in each arm are replaced by diffraction gratings (Fig. 2). The incoming wave front is separated at the beamsplitter and diffracted at the gratings, with a wavelength-dependent angle. The superposition of the two wave fronts then produces straight, parallel, and equidistant fringes with a spatial frequency depending on the wavelength of the light. The zero frequency of the fringe pattern is at the Littrow wavelength and small wavenumber changes result in fringes with discernable, low spatial frequency, which can be observed with available imaging detectors. The concept was originally proposed by Pierre Connes [2] in a configuration called "Spectromètre interférential à selection par l'amplitude de modulation (SISAM)". With the
Fig. 2 Principle design of the SHI with front and detector optics

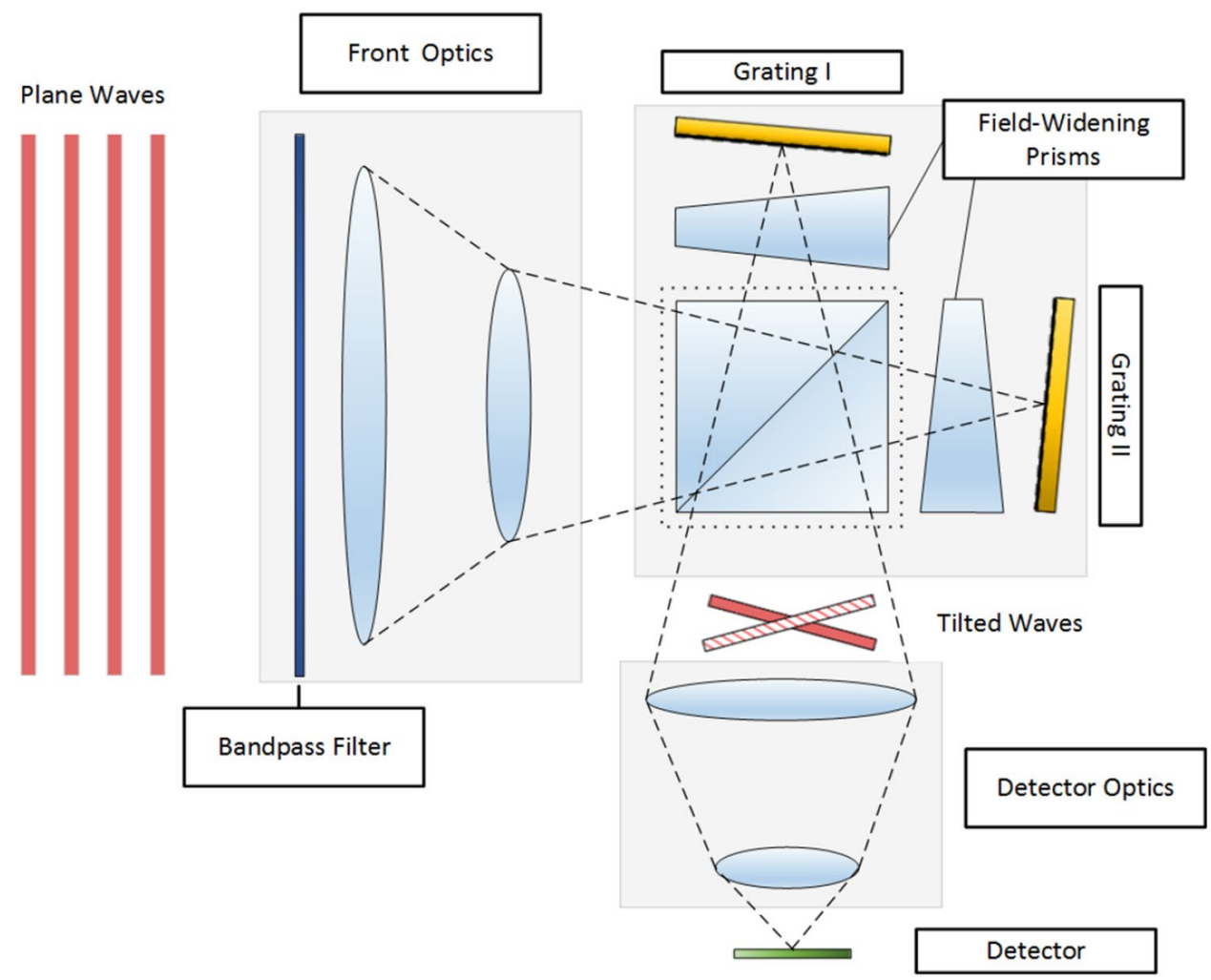


advent of imaging detectors, this idea was taken up by, e.g., Harlander and Roesler [8]; Douglas [4]; Smith and Harlander [26]; Harlander 9]; Watchorn et al. [31]; Harris et al. [12]; Roesler [23]; Englert et al. [7]; Watchorn et al. [32]; Bourassa et al. [1], and Lenzner and Diels [18]. The design of an SHI for a particular wavelength range and desired spectral resolution follows a few simple relations, which are shortly summarized to illustrate the main characteristics of this device. For a derivation of the mathematical expressions, see, e.g., Harlander et al. [10]; Cooke et al. [3]; Smith and Harlander [26], and references cited therein.

The tilt angle of the gratings with respect to the optical axis is called Littrow angle $\Theta_{L}$. Light at the Littrow wavenumber $\sigma_{\mathrm{L}}$ is returned in the same direction as the incoming path, as described by the grating equation (for diffraction order one and grating groove density $1 / \mathrm{d}$ ):

$\sigma_{L}=\frac{1}{2 d \sin \Theta_{L}}$.

Combining the intensity equation of a conventional FTS and the grating equation for small incident angles at the grating gives the SHI equation for ideal conditions, relating the incoming radiation at wavenumber $\sigma$ to the spectral density at position $\mathrm{x}$ parallel to the dispersion plane. The heterodyned fringe frequency $\kappa$ is

$\kappa=4 \tan \Theta_{L}\left(\sigma-\sigma_{L}\right)$.

The maximum resolving power of an SHI without considering apodization effects is proportional to the number of grating grooves illuminated by the incoming beam. For narrow bandwidth SHI systems, the detectable wavelength range is primarily limited by the number of detector pixel due to the Nyquist theorem.

As for conventional FTS or Fabry-Perot instruments, the acceptance angle of light for a conventional SHI is inversely proportional to its resolving power [10]. The solid angle can be increased significantly if prisms are inserted into the two interferometer arms. The prisms rotate the image of the gratings, so that they appear to be located in a common virtual plane which is oriented perpendicular to the optical axis for a wide range of incident angles. At the end, the acceptance angle of the SHI including field-widening prisms is only limited by spherical aberration for systems with small Littrow angles and astigmatism for large Littrow angles [10]. Depending on the actual design, the prisms increase the etendue or throughput of an SHI by $1-2$ orders of magnitude [10].

A general advantage of SHI is moderate alignment tolerances, because in most optical setups, the gratings are imaged onto a focal plane array. As a result, each detector pixel sees only a small area of the optical elements, so that misalignments or inaccuracies in the surface quality affect limited spatial regions on the detector, only [5, 11]. This means that the interferogram is distorted locally rather than reduced in contrast. SHI can be realized using transmitting or all-reflecting elements. SHI using dispersive elements can be built monolithically, making them very robust for harsh environments, e.g., during rocket launches.

An integral part of an SHS design is the optical filter located between the interferometer and the scene to be observed. For this instrument, a six cavity design bandpass filter with a center wavelength of $763.6 \mathrm{~nm}$ and a bandwidth of $3.4 \mathrm{~nm}$ was chosen. The front optics of a limb sounding SHI instrument adapts the angular extend of the atmospheric scene $\left(1-2^{\circ}\right)$ and the acceptance angle of the field-widened SHI $\left(5-10^{\circ}\right)$. It also images the atmosphere onto the gratings. The detector optics images the gratings onto the focal plane array. The utilization of a front optics, which has the foci on the SHI gratings, makes the system more sensitive to misalignments of the SHI than a collimated illumination, because any misalignment (such as an arm imbalance) results in shifts of foci, thus reducing the contrast of the interferogram. In addition, curved wave fronts introduce more aberrations than plane waves.

Since the performance of front- and detector optics are interdependent, they will be designed and optimized together taking the SHI performance into account. It is essential to minimize aberrations on a system level and to assure that the spatial frequencies generated by the interferometer are compatible with the modulation transfer function of the system and match pixel size and number of the focal plane array.

\section{Opto-mechanical design and assembly of an SHI}

To maintain the distances and angles between the main components of an SHI, spacers are included between the optical components. To interconnect the optical components of an SHI and to retain the distances and angles between them, spacers are included. These spacers are small glass prisms, which are located outside the optical path. A key challenge is to avoid any unwanted tilt or shift of the components with respect to each other and to maintain a well-defined bonding layer between individual components. In the initial design of an SHI as, e.g., described by Kaufmann et al. [16], a total of 18 bonds (beside the beam splitter) and the position of 15 individual components had to be controlled.

The small size of some components and their limited accessibility during the assembly procedure hampers the compliance with required tolerances. A critical step is the alignment of the gratings. The gratings and prisms are separate components to allow the use of low-cost replica gratings. Another option are GRISMs, where the grating surface is bonded directly to the side of a prism. GRISMs 
are 'high end' components and their production costs are significantly higher than all other components of the SHI. Beside opto-mechanical manufacturability, tolerancing all dimensions, distances, and angles of the components is key to obtain a system that is as tolerant as possible to misalignments and temperature variations. Bonding technologies, which are suited to assemble SHIs at room temperature, are the following:

\subsection{Adhesives}

The use of UV-cured acrylics to bond glass components is well established at low costs. The bonding layer is relatively thin, but it is not easy to assure a uniform and well-defined layer thickness. The use of several bonding layers in a stack will add up alignment tolerances. If the last bond is performed under optical/interferometric control, the misalignment can be compensated. In this case, the variable glue thickness is an advantage. Acrylic glue shrinks during curing, which affects the bonding thickness.

\subsection{Optical contacting}

This is a non-adhesive room temperature-bonding process. The surfaces are bound together by weak van der Waals molecular- or other inter-atomic forces. It is sensitive to surface particulate, chemical contamination, humidity, or mechanical and thermal stress. The surface must have a flatness in the order of $50 \mathrm{~nm}$ and must be polished. Optical contacting is a common bonding technique in the manufacturing process of optical components devices. De-bonding usually degrades surface quality. Beside a clean environment and good surface figure match, optical contacting does not require additional devices. Depending on cleanliness and surface quality, optically contacted surfaces can withstand the mechanical and thermal loads of a space instrument [7]. Otherwise, they should be complemented by additional mechanical support to fortify the bonds.

\subsection{Hydroxide catalysis}

This technique is also called silicate bonding. The chemistry involved can be considered to take place in three steps: hydration and etching, polymerization, and dehydration [14]. The $\mathrm{OH}^{-}$ions in the bonding solution etch the silica surface and liberate silicate ions, which are released into the bonding solution. The removal of $\mathrm{OH}^{-}$decreases the $\mathrm{pH}$ of the solution to a value below 11 and $\mathrm{Si}(\mathrm{OH})_{4}$ is formed, which polymerizes to form siloxane chains and water. These chains bond the two surfaces rigidly, as the water evaporates or is absorbed by the bulk material. The initial $\mathrm{pH}$ value affects the speed of the bonding process (higher $\mathrm{pH}$ gives longer setting time). Dehydration or curing takes several weeks at room temperature and can be reduced by increasing temperature. Besides etching, the silicate surfaces can also be activated by a plasma. Surface flatness has to be in the order of $50-100 \mu \mathrm{m}$ and can be relaxed further if silicate is added to the surface activator. Surfaces have to be free of particulate and chemical contamination, as for optical contacting. A moderate surface roughness avoids unwanted contacting of the surfaces before final adjustment and allows for a short alignment period until dehydration commences. Silicate bonds are very strong and stable over a large temperature range if the coefficients of thermal expansion between the two surfaces match. Hydroxide catalysis is a 'high end' bonding process for optical instruments with tight tolerances, exposed to a wide temperature range, demanding outgassing requirements and/or long duration space missions.

Beside accuracy and strength of the bonds, outgassing and lifetime are important factors to be considered in the selection process of the bonding technology. For a low Earth orbit and mission lifetimes of a few years, all of these bonding technologies are suitable. A crucial point is, whether tolerances in the assembly procedure are tight enough to omit the interferometric control of the final assembly step. Otherwise, the last bond has to be performed under interferometric control to adjust tilt and rotation of the gratings, to balance interferometer arm lengths, or to adjust the Littrow wavelength. Whereas a rotation along grating grooves changes the Littrow wavelength, a rotation around the axis which is perpendicular to grating grooves but within the grating surface tilts the two-dimensional interferogram. A rotation of the gratings perpendicular to the grating surface affects the visibility of the interferogram.

\section{Light stimulus and calibration source for an SHI instrument}

A key component in the assembly and test procedure of an SHI instrument is the illumination stimulus.

In this manuscript, we describe a light source that can be used for the bonding and assembly of an SHI and for the final calibration of the entire optical system. This includes the characterization of the visibility of the interferograms at different wavelengths, the flat field of the instrument, and the instrumental line shape. It can be used to verify the Littrow wavelength and the absolute radiometric responsivity as well if suitable detectors to quantify the radiant flux are available.

A key requirement to perform these calibrations using the entire instrument is to generate a light stimulus which is as similar as possible to the final observing geometry. In space, the SHI instrument is illuminated by plane waves, which are focused by the front optics onto the gratings. This means that the SHI is illuminated by waves with strongly curved wave fronts. The optical system is designed in such 
a way that optical aberrations are minimized in this specific configuration. If the incident radiation at the front optics is not properly collimated, the waves will experience different aberrations, which will lead to a different visibility of the interferograms and to a potential miscalibration of the system.

To characterize the visibility of the system, a tunable (laser) light source is most suitable to cover all spatial frequencies recorded by the instrument. The ratio of the modulated and the total signal defines the visibility of the system, which typically decreases towards high spatial frequencies due to optical aberrations. The instrument line shape can be obtained from the same measurements. Nonuniformities of the system caused by different sensitivities of detector elements, inhomogeneities within optical components, or any kind of misalignment of the optical components including the SHI can be determined using this light stimulus as well. The methodology used is the "balanced arm flat-fielding approach" Englert and Harlander [6], where the SHI

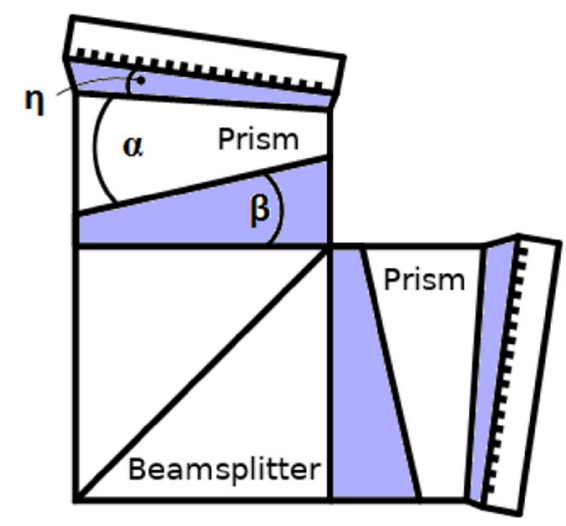

Fig. 3 Top view of SHI [17]. The blue-shaded components are spacers, which are not in the light path. $\alpha, \beta$, and $\eta$ are prism apex angle and spacer angles, respectively arms are blocked one by one to measure non-modulated intensities.

Thus, two major requirements have to be fulfilled by the illumination stimulus. At first, a homogeneous scene has to be virtually placed to infinity, and second, the wave fronts exiting the light stimulus have to be as plane as possible over the full field-of-view of the instrument. To generate a homogeneous top hat profile, a plane diffuser in front of an Ulbricht sphere or a setup of one or more micro lens arrays (MLAs) forming a Köhler integrator [15] can be used. Afterwards, the infinite conjugate of this scene can be achieved by a suitable telescope. For the space application described here, the Köhler illumination concept was chosen, because the overall size of the setup is small and fits well into a thermal vacuum chamber to test the SHI instrument at various temperatures and under vacuum conditions (Fig. 3).

The principal setup can be divided into the laser light source, a de-speckle device, a homogeneous scene generator (HSG), and an imaging optics (Fig. 4).

To break the spatial coherence of the laser light, a rotating diffuser [28] is used. To illuminate the diffuser, a fore optics (finite-finite conjugate configuration) images the laser light emitted from a fiber coupler onto a well-defined spot. To obtain a homogenous flat-top profile, a configuration of two lenses, two identical MLAs, and a second rotating diffuser is used.

The front focal plane of the lens just before the first MLA coincides with the back side of the first rotating diffuser. In this configuration, the first MLA is illuminated with collimated light so that each sub-aperture of the MLA forms a small image at the focal point. The divergence of the beam is given by the spot size on the second diffuser. To avoid overfilling, the distance between the two MLAs is adjusted in such a way that individual beamlets are smaller than the lenslet size of the second MLA. Typically, the second MLA is placed close to the focal plane of the first one. The

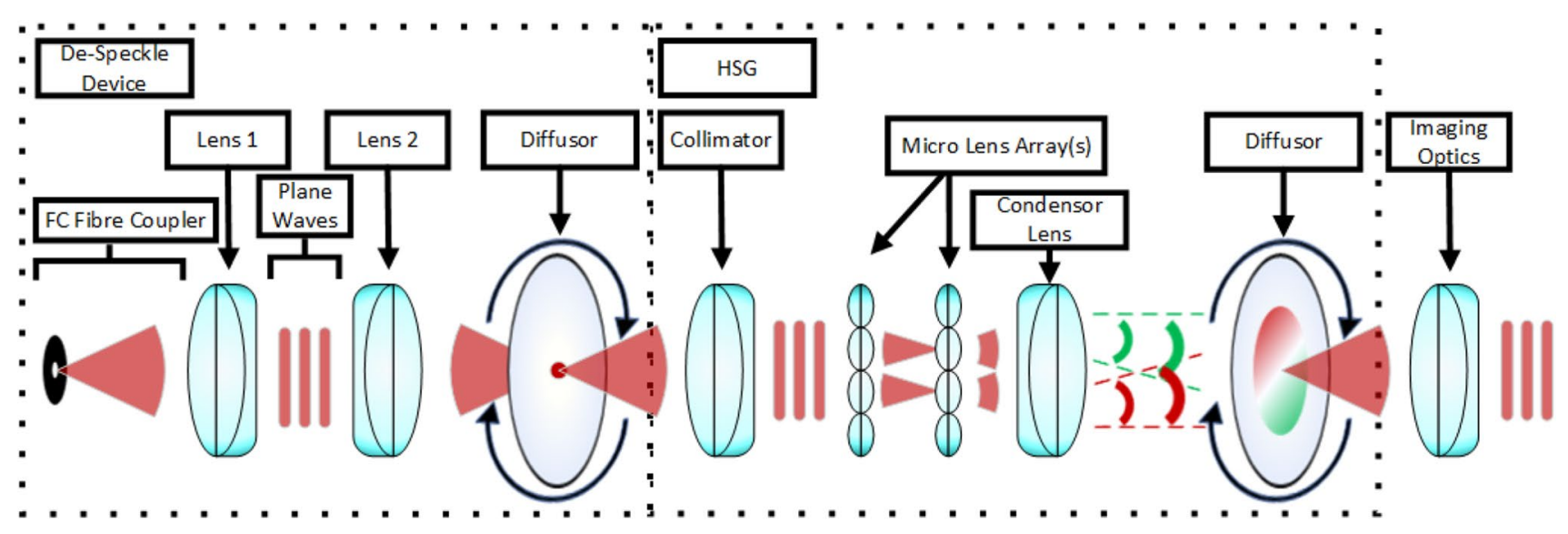

Fig. 4 Light stimulus and calibration setup for an SHI instrument 


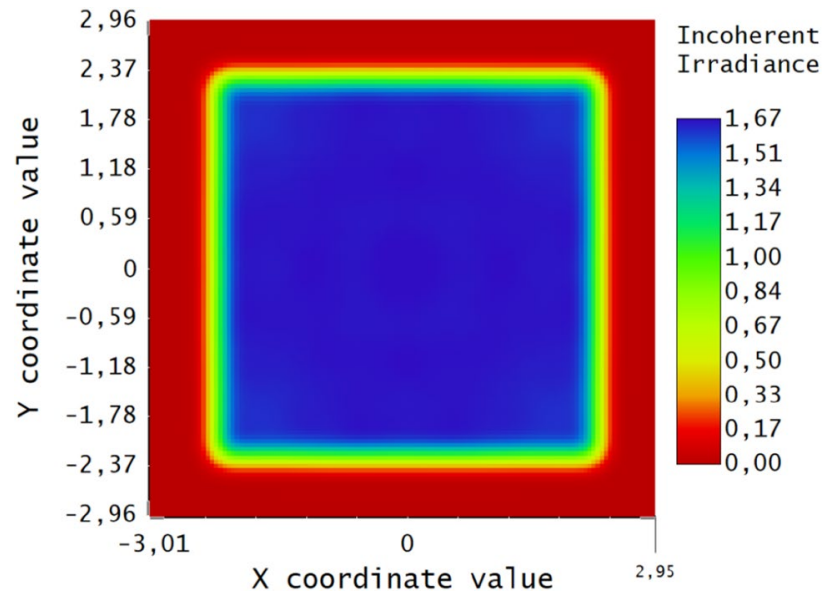

Fig. 5 Simulated homogeneous scene as seen at the second diffuser

selection of suitable MLAs (pitch, focal length, etc.) and adjacent lenses is described, e.g., in Voelkel and Weible [30]. Dependent on the pitch $p$, the focal length $f_{L A}$ of the MLAs, and the focal length $\mathrm{f}_{\mathrm{FL}}$ of the following condenser lens, the size A of the illuminated flat-top profile in the object plane at the second diffuser follows [30]:

$\mathrm{A}=\mathrm{p} \times f_{\mathrm{FL}} / f_{\mathrm{LA}}$.

In the third section of the optical setup, the flat-top profile at the diffuser is virtually placed at the infinite conjugate which mimics the instruments viewing geometry of the atmosphere.

As an example, two MLAs with a pitch of $300 \mu \mathrm{m}$ and a focal length of $8.7 \mathrm{~mm}$ were simulated using the optical ray-tracing software (Fig. 5). The condenser lens' focal length is $150 \mathrm{~mm}$. The simulation shows a well-defined top hat profile, whose actual area is with 1-2 mm smaller than calculated using the formula given above.

Open Access This article is distributed under the terms of the Creative Commons Attribution 4.0 International License (http://creativeco mmons.org/licenses/by/4.0/), which permits unrestricted use, distribution, and reproduction in any medium, provided you give appropriate credit to the original author(s) and the source, provide a link to the Creative Commons license, and indicate if changes were made.

\section{References}

1. Bourassa, A.E., Langille, J., Solheim, B., Degenstein, D., Dupont, F.: The spatial heterodyne observations of water (SHOW) instrument for high resolution profiling in the upper troposphere and lower stratosphere, in light, energy and the environment. Opt. Soc. Am. (2016). https://doi.org/10.1364/FTS.2016.FM3E.1

2. Connes, P.: Spectromètre interférential à selection par l'amplitude de modulation. J. Phys. Radium 19(3), 215-222 (1958)

3. Cooke, B.J., Smith, B.W., Laubscher, B.E., Villeneuve, P.V., Briles, S.D.: Analysis and system design framework for infrared spatial heterodyne spectrometers. Proc. SPIE 3701, 167-192 (1999)

4. Douglas, N.G.: Heterodyned holographic spectroscopy. Publ. Astron. Soc. Pac. 109(732), 151 (1997)

5. Englert, et al.: Correction of Phase Distortion in Spatial Heterodyne Spectroscopy. Appl. Opt. 43, 6680-6687 (2004). https://doi. org/10.1364/AO.43.006680

6. Englert, C.R., Harlander, J.M.: Flatfielding in spatial heterodyne spectroscopy. Appl. Opt. 45, 4583-4590 (2006). https://doi. org/10.1364/AO.45.004583

7. Englert, C.R., Stevens, M.H., Siskind, D.E., Harlander, J.M., Roesler, F.L.: Spatial Heterodyne Imager for Mesospheric Radicals on STPSat-1. J.Geophys.Res. Atmos. 115, D20306 (2010). https://doi.org/10.1029/2010JD014398

8. Harlander, J.M., Roesler, F.L.: Spatial heterodyne spectroscopy: a novel interferometric technique for ground-based and space astronomy. Proc. SPIE 1235, 622-634 (1990)

9. Harlander, J.M.: Spatial Heterodyne Spectroscopy: Interferometric Performance at any Wavelength Without Scanning. Ph.D. dissertation, The University of Wisconsin-Madison (1991)

10. Harlander, J.M., Reynolds, R.J., Roesler, F.L.: Spatial heterodyne spectroscopy for the exploration of diffuse interstellar emission lines at far-ultraviolet wavelengths. Astrophys. J. 396, 730-740 (1992)

11. Harlander, et al.: Field-Widened Spatial Heterodyne Spectroscopy: Correcting for Optical Defects and New Vacuum Ultraviolet Performance Tests. Proc. SPIE 2280, 310-319 (1994)

12. Harris, W.M., Roesler, F.L., Harlander, J., Ben-Jaffel, L., Mierkiewicz, E., Corliss, J., Oliversen, R.J.: Applications of reflective spatial heterodyne spectroscopy to UV exploration in the solar system. Proc. SPIE 5488, 5488 (2004). https://doi. org/10.1117/12.553107

13. Huba, J., Schunk R. Khazanov G. (eds): Modeling the ionosphere-thermosphere system, Published by the American Geophysical Union as part of the Geophysical Monograph Series, 201, Wiley, Chichester, UK (2014). https://doi. org/10.1002/9781118704417

14. Iler, R.: The Chemistry of Silica. Interscience, New York (1979)

15. Köhler, A.: Ein neues Beleuchtungsverfahren für mikrophotographische Zwecke. Zeitschrift für wissenschaftliche Mikroskopie und für Mikroskopische Technik. 10(4), 433-440 (1893)

16. Kaufmann, M., Blank, J., Guggenmoser, T., Ungermann, J., Engel, A., Ern, M., Friedl-Vallon, F., Gerber, D., Grooß, J.U., Guenther, G., Höpfner, M., Kleinert, A., Kretschmer, E., Latzko, T.H., Maucher, G., Neubert, T., Nordmeyer, H., Oelhaf, H., Olschewski, F., Orphal, J., Preusse, P., Schlager, H., Schneider, H., Schuettemeyer, D., Stroh, F., Suminska-Ebersoldt, O., Vogel, B., Volk, M.C., Woiwode, W., Riese, M.: Retrieval of three-dimensional small-scale structures in upper-tropospheric/lower-stratospheric composition as measured by GLORIA. Atmos. Meas. Tech. 8, 81-95 (2015). https://doi.org/10.5194/amt-8-81-2015

17. Kaufmann, M., Olschewski, F., Mantel, K., Solheim, B., Shepherd, G., Deiml, M., Liu, J., Song, R., Chen, Q., Wroblowski, O., Wei, D., Zhu, Y., Wagner, F., Loosen, F., Froehlich, D., Neubert, T., Rongen, H., Knieling, P., Toumpas, P., Shan, J., Tang, G., Koppmann, R., Riese, M.: A highly miniaturized satellite payload based on a spatial heterodyne spectrometer for atmospheric temperature measurements in the mesosphere and lower thermosphere. Atmos. Meas. Tech. 11, 3861-3870 (2018). https://doi. org/10.5194/amt-11-3861-2018

18. Lenzner, M., Diels, J.-C.: Concerning the spatial heterodyne spectrometer. Opt. Express 24(2), 1829-1839 (2016)

19. Miladinovich, D.S., Datta-Barua, S., Bust, G.S., Makela, J.J.: Assimilation of thermospheric measurements for 
ionosphere-thermosphere state estimation. Radio Sci. 51, 18181837 (2016). https://doi.org/10.1002/2016RS006098

20. Oberheide, J., Shiokawa, K., Gurubaran, S., Ward, W.E., Fujiwara, H., Kosch, M.J., Makela, J.J., Takahashi, H.: The geospace response to variable inputs from the lower atmosphere: a review of the progress made by Task Group 4 of CAWSES-II. Prog. Earth Planet. Sci. 2, 2 (2015)

21. Olschewski, F., Kaufmann, M., Mantel, K., Neubert, T., Rongen, H., Riese, M., Koppmann, R.: AtmoCube A1: Airglow measurements in the mesosphere and lower thermosphere by spatial heterodyne interferometry. J. Appl. Remote Sens. 13(2), 024501 (2019)

22. Ortland, D.A., Hays, P.B., Skinner, W.R., Yee, J.-H.: Remote sensing of mesospheric temperature and $\mathrm{O}_{2}\left({ }^{1} \Sigma\right)$ band volume emission rateswith the high-resolution Doppler imager. J. Geophys. Res. 103, 1821-1835 (1998)

23. Roesler, F.L.: An overview of the shs technique and applications, in: fourier transform spectroscopy/hyperspectral imaging and sounding of the environment. Opt. Soc. Am. (2007). https://doi. org/10.1364/FTS.2007.FTuC1

24. Scherliess, L., Thompson, D.C., Schunk, R.W.: Data assimilation models: A 'New' Tool for Ionospheric Science and Applications. (2011). https://doi.org/10.1007/978-94-007-0501-2_18

25. Sheese, PE., Llewellyn, E.J., Gattinger, R.L., Bourassa, A.E., Degenstein, D.A., Lloyd, N.D., McDade, I.C.: Temperatures in the upper mesosphere and lower thermosphere from OSIRIS observations of $\mathrm{O}_{2}$ A-band emission spectra. Can. J. Phys. 88, 919-925 (2010). https://doi.org/10.1139/p10-093

26. Smith, B.W., Harlander, J.M.: Imaging spatial heterodyne spectroscopy: theory and practice. Proc. SPIE 3698, 925-932 (1999)

27. Song, R., Kaufmann, M., Ern, M., Ungermann, J., Liu, G., Riese, M.: Three-dimensional tomographic reconstruction of atmospheric gravity waves in the mesosphere and lower thermosphere (MLT). Atmos. Meas. Tech. 11, 3161-3175 (2018). https://doi. org/10.5194/amt-11-3161-2018
28. Stangner, T., Zhang, H., Dahlberg, T., Wiklund, K., Andersson, M.: Step-by-step guide to reduce spatial coherence of laser light using a rotating ground glass diffuser. Appl. Opt. 56, 5427-5435 (2017)

29. Ungermann, J., Blank, J., Dick, M., Ebersoldt, A., Friedl-Vallon, F., Giez, A., Guggenmoser, T., Höpfner, M., Jurkat, T., Kaufmann, M., Kaufmann, S., Kleinert, A., Krämer, M., Latzko, T., Oelhaf, H., Olchewski, F., Preusse, P., Rolf, C., Schillings, J., SuminskaEbersoldt, O., Tan, V., Thomas, N., Voigt, C., Zahn, A., Zöger, M., Riese, M.: Level 2 processing for the imaging Fourier transform spectrometer GLORIA: Derivation and validation of temperature and trace gas volume mixing ratios from calibrated dynamics mode spectra. Atmos. Meas. Tech. 8, 2473-2489 (2015). https:// doi.org/10.5194/amt-8-2473-2015

30. Voelkel, R., Weible, K.J.: Laser beam homogenizing: Limitations and constraints. Proc. SPIE 5, 5-9 (2008). https://doi. org/10.1117/12.799400. (Optical Fabrication, Testing, and Metrology III)

31. Watchorn, S., Roesler, F.L., Harlander, J.M., Jaehnig, K.P., ReynoldsRJ, Sanders W.T.: Development of the spatial heterodyne spectrometer for vuv remote sensing of the interstellar medium. Proc. SPIE 4498, 284-296 (2001)

32. Watchorn, S., Roesler, F.L., Harlander, J., Jaehnig, K.P., Reynolds, R.J., Sanders, W.T.: Evaluation of payload performance for a sounding rocket vacuum ultraviolet spatial heterodyne spectrometer to observe C IV $\lambda \lambda 1550$ emissions from the Cygnus Loop. Appl. Opt. 49, 3265-3273 (2010). https://doi.org/10.1364/ AO.49.003265

Publisher's Note Springer Nature remains neutral with regard to jurisdictional claims in published maps and institutional affiliations. 